\title{
COMPARING THE MAIN OSCILLATION CHARACTERISTICS IN THE SOLAR CHROMOSPHERE AND MAGNETOSPHERE BASED ON STUDIES MADE AT ISTP SB RAS
}

\author{
A.A. Chelpanov \\ Institute of Solar-Terrestrial Physics SB RAS, \\ Irkutsk, Russia, chelpanov@iszf.irk.ru

\section{M.A. Chelpanov} \\ Institute of Solar-Terrestrial Physics SB RAS, \\ Irkutsk, Russia,max_chel@iszf.irk.ru
}

\author{
N.I. Kobanov \\ Institute of Solar-Terrestrial Physics SB RAS, \\ Irkutsk, Russia,kobanov@iszf.irk.ru \\ R.T. Sotnikova \\ Irkutsk State University, \\ Irkutsk, Russia,rsotnikova@bk.ru
}

\begin{abstract}
The aim of this paper is to structure and extend the knowledge of solar chromospheric sources of oscillations in the solar wind and their relationships with pulsations registered in the magnetosphere. We compare the oscillation spectra that we observe using instruments of the Institute of Solar-Terrestrial Physics in different chromospheric structures with those observed in the solar wind and magnetosphere. We explore the possibility that the observed periodic variations of the chromospheric line widths can be interpreted as torsional Alfvén wave manifestation - this mode can propagate long distances without dissipating in the interplanetary
\end{abstract}

space; it can penetrate into Earth's magnetosphere directly or due to processes occurring at the plasmapause. We emphasize the similarities in the oscillation characteristics observed in different media, the similarities in the parameters of the media themselves and the processes developing in them. We believe that similar approaches can be applied to studying these media.

Keywords: solar faculae, sunspots, MHD waves, magnetosphere.

\section{INTRODUCTION}

Magnetohydrodynamic (MHD) waves are thought to be one of the mechanisms of energy transport in the heliosphere. They occur in all its regions, from the photosphere to Earth's atmosphere.

In all layers of the solar atmosphere there is a rich variety of periods and MHD modes. A large amount of observational data has been accumulated for objects of various types. In this work, we generally use the waves that are observed in the upper chromosphere because they are more likely to influence processes in the solar wind (SW) in contrast, for example, to photospheric oscillations, which are known to be trapped sometimes in a limited range of heights without penetrating into the upper atmosphere.

Oscillations in the upper atmosphere and magnetosphere have also been studied for a long time. One of the key mechanisms leading to the generation of ultralow-frequency (ULF) waves in Earth's magnetosphere is the mechanism of direct penetration of waves from SW through the magnetopause, which was first proposed by Guglielmi, Troitskaya [1973], [Mazur, 2010; Greenstadt et al., 1983]. In [Kessel, 2008; Takahashi, Ukhorskiy, 2007, 2008], SW pressure fluctuations are considered as the main source of longperiod pulsations in the magnetosphere.

We examine properties of the oscillations observed in the chromosphere and SW to find a link between them. This work is based on the observation results from previous research carried out at the Institute of Solar-Terrestrial Physics of Siberian Baranch of Russian Academy of Sciences (ISTP SB RAS) on geomagnetic oscillations associated with MHD oscillations in the upper solar chromosphere.

We focus on the studies conducted for a number of structures of the solar atmosphere in different spectral lines formed in a wide range of heights.

We study the oscillation characteristics of line-ofsight velocity, intensity, and profile width. Methods of measurements are described in [Kobanov, 1985, 2001]. In this work, we analyze the possible effects of torsional Alfvén waves, characterized by the rotation of magnetic tube segments. Alfvén waves can propagate over long distances with minimal dissipation, traveling from the chromosphere through the corona in SW and reaching Earth. On the other hand, Cranmer and van Ballegooijen [2005] have shown that the transition region can reflect a large number of Alfvén waves in a wide range of periods. In interplanetary space, the reflection is essential only for periods of the order of tens of hours or more. In our previous studies, we observed periodic variations in the width of chromospheric lines, which can be interpreted as manifestations of torsional Alfvén waves in different structures of the solar atmosphere. The next step is to trace the dynamics of these waves in SW and Earth's magnetosphere after they have been generated in the solar chromosphere.

Below we provide a brief description of the characteristics of periodic oscillations in the Sun and Earth's magnetosphere, which were found in our observational data.

\section{OSCILLATIONS ON THE SUN}

Oscillations in the solar chromosphere manifest themselves in a wide range of periods. Characteristics of the oscillations are known to be determined by the mag- 
netic field strength and topology in solar structures: sunspots, faculae, coronal holes. ISTP SB RAS has extensive experience of research into oscillations in these objects, which we have used for this paper. We pay special attention to observational signatures of Alfvén waves in objects of interest. Observing Alfvén waves on the Sun is a difficult task because they are incompressible waves and hence cannot produce intensity variations. As a result, Alfvén waves cannot be recorded in emission variations [Nakariakov, Verwichte, 2005]. They have been recorded so far on the basis of indirect signatures or directly in spicules at the limb with the use of high-resolution data. One of their signatures is periodic variations in spectral line profile width. De Pontieu et al. [2015] have shown that these variations do not depend on the spatial resolution of the instrument in use.

It should be noted that until an alternative convincing explanation for the abnormally high amplitudes of the chromospheric spectral line profile width variations is found, the version of torsional Alfvén waves in the solar chromosphere has the right to exist.

\section{Oscillations in coronal holes}

Coronal holes are the source of high-speed SW streams, so they become an important object of research into wave propagation and generation in the Sun-Earth system. Coronal holes feature large-scale magnetic fields, which penetrate the solar atmosphere from its lower layers to the corona. These magnetic fields can act as waveguides for MHD waves. From observations of 15 coronal holes, Kobanov and Sklyar [2007] have found pronounced oscillations in three- and five-minute ranges. In the five-minute range, they found signs of propagating waves. Kobanov et al. [2003] observed LOS velocity oscillations with frequencies of $1 \mathrm{mHz}$ and lower along with three- and five-minute oscillations above coronal holes.

Anfinogentov et al. [2010], using the $171 \AA$ coronal line, observed longitudinal and transverse oscillations predominantly with $15-30$ min periods in loops located in a coronal hole. These oscillations were caused by eruption at a bright point of the coronal hole.

Zubkova et al. [2014] have shown that the amplitude of chromospheric line profile half-width oscillations observed in 10 coronal holes are too large to be ex- plained only by temperature variations. Table 1 lists amplitudes of half-width oscillations for observation series recorded in coronal holes. Such oscillations may indicate the presence of torsional Alfvén waves. Frequencies of the half-width oscillations were distributed in the $1.5-6.5 \mathrm{mHz}$ range with the most prominent peaks in the $3.5-5.5 \mathrm{mHz}$ range (Figure 1). Oscillation power in this frequency range increases in structural chromospheric elements within coronal holes. This increase, according to the authors, is due to the concentration of vertical magnetic fields in the vicinity of the chromospheric network. When analyzing characteristics of these oscillations, we should, however, take into account that Alfvén waves propagating in an inhomogeneous MHD medium of the solar atmosphere promote phase mixing, which leads to disruption of monochromaticity and transformation of primary signals [Shestov et al., 2017].

\section{Oscillations in faculae}

Faculae feature five-minute oscillations in signals of LOS velocities both in the photosphere and in the chromosphere [Kobanov et al., 2013a], although the spatial distribution of the oscillations in faculae is inhomogeneous. In LOS velocity signals, frequencies are higher in regions of intense magnetic field (magnetic hills) and lower closer to the boundaries of faculae [Chelpanov et al., 2015; Chelpanov et al., 2016b]. Kobanov and Pulyaev [2007] have found signs of propagating waves above magnetic hills. Kobanov et al. [2011] have analyzed phase relationships between the five-minute oscillations at different heights and have concluded that the waves propagate both upward and downward, thus returning a part of the wave energy from the chromosphere to the photosphere. We have identified a number of cases where chromospheric line half-width oscillations in faculae were not accompanied by intensity or velocity oscillations [Chelpanov et al., 2016a]. Figure 2 gives examples of half-width oscillation spectra in faculae. Spectral line profile half-width oscillation amplitudes in faculae are presented in Table 2. These examples do not, however, show the relationship between the half-width oscillation amplitude and the center-to-limb position on the solar disk. This can be explained by the

\begin{tabular}{|c|c|c|c|c|}
\hline $\begin{array}{c}\text { Series date and time, } \\
\text { UT }\end{array}$ & Spectral line & $\begin{array}{c}\text { Location } \\
\text { on the solar disk }\end{array}$ & $\begin{array}{c}\text { Duration, } \\
\text { min }\end{array}$ & $\begin{array}{c}\text { Average peak-to-peak } \\
\text { amplitude, } m \AA\end{array}$ \\
\hline Aug 4, 2005, 06:26 & $\mathrm{H} \alpha$ & $32 \mathrm{~S} 4 \mathrm{~W}$ & 43 & $22.98(52)$ \\
\hline May $29,2010,05: 31$ & $\mathrm{H} \alpha$ & North pole & 63 & $23.21(45.6)$ \\
\hline May 29, 2010, 07:09 & $\mathrm{H} \alpha$ & North pole & 63 & $24.15(50.5)$ \\
\hline Jun 16, 2010, 04:34 & $\mathrm{H} \alpha$ & $25^{\circ} \mathrm{N} 35^{\circ} \mathrm{W}$ & 170 & $27.01(78)$ \\
\hline Jul 06, 2010, 10:21 & $\mathrm{H} \alpha$ & $7^{\circ} \mathrm{N} 13^{\circ} \mathrm{W}$ & 83 & $24.86(55.6)$ \\
\hline Apr 10, 2011, 07:27 & $\mathrm{H} \alpha$ & $25^{\circ} \mathrm{S} 15^{\circ} \mathrm{W}$ & 85 & $20.92(63.3)$ \\
\hline Apr 12, 2011, 01:21 & $\mathrm{H} \alpha$ & $56^{\circ} \mathrm{S} 2^{\circ} \mathrm{E}$ & 83 & $37.26(88)$ \\
\hline Sep 18, 2011, 05:36 & $\mathrm{H} \alpha$ & $30^{\circ} \mathrm{S} 52^{\circ} \mathrm{E}$ & 67 & $33.14(98.5)$ \\
\hline Sep 18, 2011, 07:43 & $\mathrm{H} \alpha$ & $30^{\circ} \mathrm{S} 52^{\circ} \mathrm{E}$ & 82 & $27.87(50.6)$ \\
\hline Sep 19, 2011, 01:57 & $\mathrm{H} \alpha$ & $30^{\circ} \mathrm{S} 40^{\circ} \mathrm{E}$ & 65 & $35.80(59.25)$ \\
\hline Aug 11, 2005, 02:54 & Ca II $8542 \AA$ & $4^{\circ} \mathrm{N} 47^{\circ} \mathrm{E}$ & 57 & $46.3(83.3)$ \\
\hline Aug $11,2005,04: 00$ & Ca II $8542 \AA$ & $60^{\circ} \mathrm{N} 1^{\circ} \mathrm{E}$ & 41 & $45(103.5)$ \\
\hline Aug 17, 2005, 07:12 & Ca II $8542 \AA$ & $0^{\circ} \mathrm{N} 37^{\circ} \mathrm{W}$ & 81 & $70(115.5)$ \\
\hline Sep 29, 2005, 03:29 & Ca II $8542 \AA$ & $35^{\circ} \mathrm{S} 0^{\circ} \mathrm{E}$ & 52 & $72.4(136.9)$ \\
\hline May $28,2010,06: 16$ & Ca II $8542 \AA$ & North pole & 120 & $44.75(122.9)$ \\
\hline
\end{tabular}

Table 1 
Table 2

\begin{tabular}{|c|c|c|c|}
\hline $\begin{array}{c}\text { Series date and duration, } \\
\text { min }\end{array}$ & $\begin{array}{c}\text { Spectral } \\
\text { line }\end{array}$ & \multicolumn{2}{|c|}{$\begin{array}{c}\text { Average peak-to-peak amplitude } \\
\text { of half-width, } \AA\end{array}$} \\
\cline { 3 - 4 } & & $2.5-4.5 \mathrm{mHz}$ & $1.0-1.9 \mathrm{mHz}$ \\
\hline Jul 1, 2003, 42 & $\mathrm{H} \alpha 6563 \AA$ & $9-21$ & 23 \\
\hline Sep 12, 2003, 52 & $\mathrm{H} \alpha 6563 \AA$ & $20-25$ & 35 \\
\hline Jul 27, 2005, 67 & $\mathrm{H} \alpha 6563 \AA$ & $20-50$ & 40 \\
\hline Sep 24, 2005, 84 & $\mathrm{H} \alpha 6563 \AA$ & $20-30$ & - \\
\hline \multirow{2}{*}{ Sep 21, 2012, 100 } & $\mathrm{HeI} \mathrm{10830 \AA}$ & 40 & - \\
\cline { 2 - 4 } & $\mathrm{H} \alpha 6563 \AA$ & $35-45$ & $40-60$ \\
\hline May 1, 2013,47 & $\mathrm{H} \alpha 6563 \AA$ & $30-35$ & 45 \\
\hline \multirow{2}{*}{ Aug 25, 2013,75 } & $\mathrm{HeI} 10830 \AA$ & $40-50$ & 70 \\
\cline { 2 - 4 } & $\mathrm{H} \alpha 6563 \AA$ & $30-35$ & 55 \\
\hline \multirow{2}{*}{ Aug 28, 2013, 158} & $\mathrm{HeI} 10830 \AA$ & 45 & 50 \\
\cline { 2 - 4 } & $\mathrm{H} \alpha 6563 \AA$ & 25 & \\
\hline
\end{tabular}

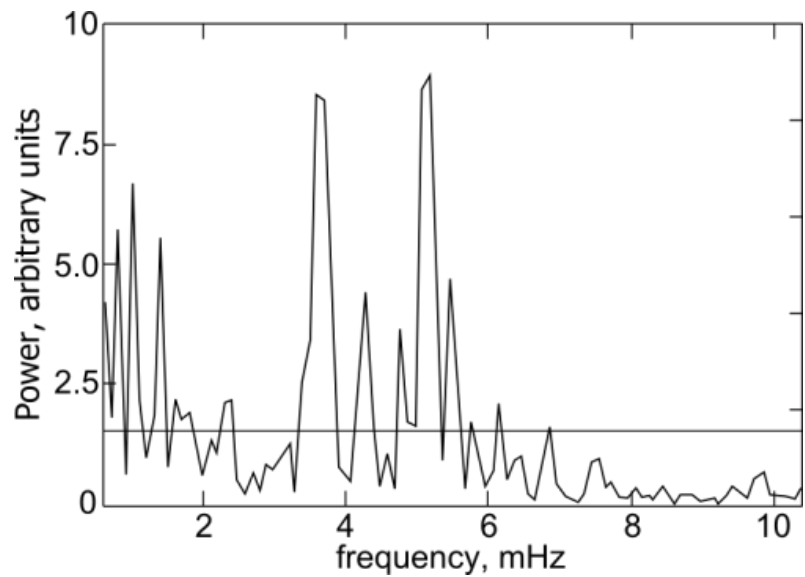

Figure 1. Typical $\mathrm{H} \alpha$ profile half-width oscillation spectrum in a coronal hole. The main oscillation power is distributed in the $1.5-6.5 \mathrm{mHz}$ range

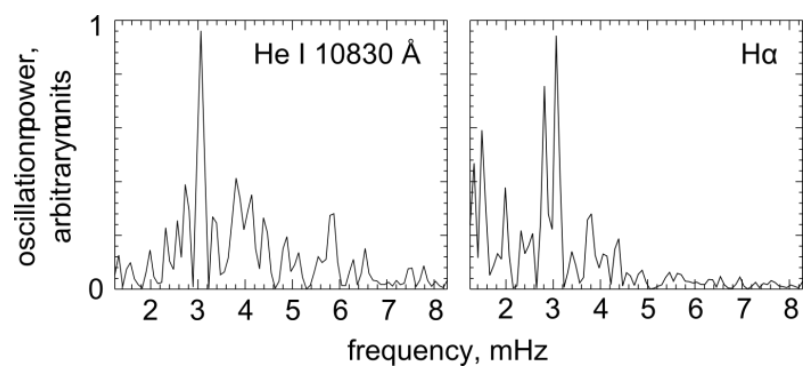

Figure 2. Chromospheric line profile half-width oscillation spectra in a facula

the magnetic field configuration in faculae - the faculae area is densely populated with magnetic fields of different slopes, which theoretically makes it possible to observe torsional Alfvén waves at any point of the solar disk.

\section{Active regions}

Unlike faculae, there are oscillations with different periods in sunspot umbrae at different heights: five-minute oscillations dominate in the photosphere; and three-minute oscillations, in the chromosphere. Figure 3 presents typical spectra observed in the photosphere and chromosphere of sunspot umbrae. Over the entire surface of the sunspot and in its vicinity, the observed oscillation range is much wider. Oscillation power distributions in sunspot umbrae, penumbrae, and their vicinities have shown that the periods increase from inner umbrae to penumbrae and further through the outer boundaries in the vicinity of the sunspot, where pronounced 10-12 min oscillations prevail [Kobanov, 2000b; Kobanov et al., 2013b; Kobanov et al., 2015].

Kolobov et al. [2016] have examined a circular region in the inner penumbra, where five-minute oscillations prevail in a wide range of heights, from the photosphere to the transition region (Figure 4).

The authors believe that the slope and strength of the magnetic field in this region determine conditions for propagation of five-minute oscillations between layers of the solar atmosphere.

Sych et al. [2009] have examined the possible connection between three-minute oscillations in sunspots and similar oscillations observed in flare light curves. The authors assumed that three-minute oscillations propagate from sunspots to flares, inducing there an energy release with this period.

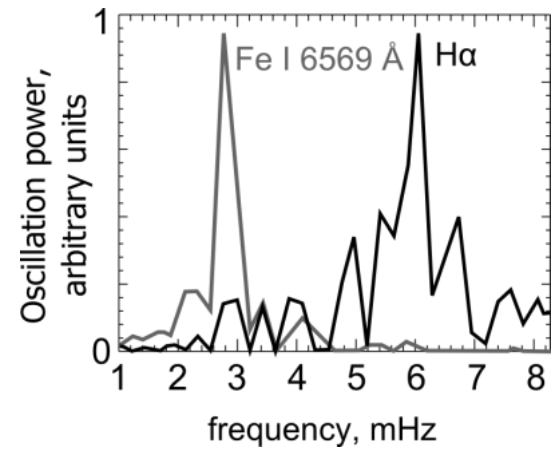

Figure 3. Umbral line-of-sight velocity oscillation spectra in the photosphere (Fe I $6569 \AA)$ and chromosphere $(\mathrm{H \alpha})$

$1700 \AA$

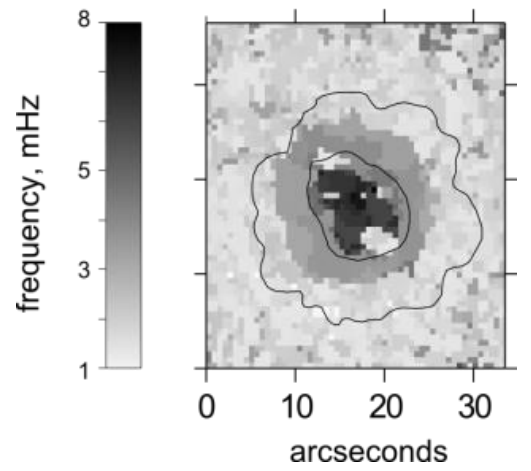

Figure 4. Distribution of dominant frequencies observed in a sunspot and its vicinity 
Sych et al. [2012] have also studied three-minute oscillations observed in EUV channels and in a microwave range above sunspots. When analyzing their frequency characteristics, the authors found that the oscillation period can gradually change during one oscillation train. Two mechanisms were proposed: evolution of subphotospheric oscillation sources in the vicinity of sunspot and separation of pulses in magnetic tubes with different physical conditions.

One of the recent discoveries in oscillations in active regions is undamped transverse oscillations of coronal loops [Anfinogentov et al., 2015]. Periods of these oscillations can vary from 1 to $10 \mathrm{~min}$, which corresponds approximately to loop eigenoscillation periods. They occur in almost all active regions and are likely to result from the interaction of loops with surrounding quasistationary streams [Nakariakov et al., 2016b].

\section{OSCILLATIONS IN THE MAGNETOSPHERE}

Geomagnetic waves, or pulsations, can be regularly observed in any part of Earth's magnetosphere. Stable periodic and quasiperiodic pulsations are usually referred to as Pc, or continuous pulsations. Compared to other similar pulsations, Pc5 waves (periods of 150 to $600 \mathrm{~s}$ ) have much higher amplitudes. At high latitudes, they can reach hundreds of nanotesla even under moderately disturbed geomagnetic conditions.

Pc5 waves can have both internal and external magnetospheric sources. The azimuthal structure of oscillations is often considered as an indicator of their origin: waves generated within the magnetosphere usually feature a high azimuthal wave number $m$; waves with sources in SW or at the magnetopause usually exhibit low $m$ values because waves with high $m$ cannot effectively penetrate into the magnetosphere [Guglielmi, Potapov, 1984; Leonovich et al., 2015].

Long-period pulsations of another type - Pi2 - are irregular pulsations with dominant periods of 40 to $150 \mathrm{~s}$. They represent isolated wave packets and are more common than Pc5 [Lipko et al., 2002]. Pi2 are often observed in 5-10 min series. They usually occur during geomagnetic storms and substorms. They can accompany all phases of magnetic storms generated by continuous substorm activity [Zolotukhina et al., 2000]. At midlatitudes, Pi2 pulsations can be observed in a wide longitude sector, including the dayside magnetosphere. The region of Pi2 surface observation often corresponds approximately to the plasmapause footpoint. Rakhmatulin et al. [2000], using a network of mid-latitude observatories, have determined a stable maximum of Pi2 occurrence around noon. In the nightside magnetosphere, however, high-latitude pulsations predominate over mid-latitude ones. The presence of the secondary longitudinal maximum and the differences between Pi2 generation patterns in different regions confirm the assumption about multiple sources of these pulsations [Rakhmatulin, 2009].

The hypothesis about penetration of SW-driven oscillations into the magnetosphere was made in the 1970s [Guglielmi, 1972; Troitskaya et al., 1971]; then it was developed and supported by many researchers [Potapov,
Mazur, 1994]. The most frequently discussed mechanism of externally generated waves is the KelvinHelmholtz instability forming at the magnetopause under the influence of high-speed SW [Lee et al., 1981; Mishin, Tomozov, 2016]. While the magnetopause is an integral part of the magnetosphere, surface waves propagating in it and causing internal magnetospheric oscillations are also external sources since they are a direct consequence of the interaction between the magnetosphere and SW inhomogeneities [Yumoto, 1988]. Fast magnetosonic (FMS) waves excited by this process propagate into the magnetosphere and generate Alfvén waves on resonance magnetic shells, where the frequency of local Alfvén oscillations coincides with the frequency of the FMS wave exciting these oscillations [Mishin, 1981, 1993; Dmitrienko, 2010]. This hypothesis is supported by many features of magnetospheric waves: peculiarities of polarization pattern, preferential antisunward propagation [Olson, Rostoker, 1978], dependence of pulsation observation probability on SW speed [Nosé et al., 1995], etc.

Among other scenarios, a direct penetration of ULF waves from an interplanetary medium into the magnetosphere is often discussed [Mazur, 2010; Stephenson, Walker, 2002]. Potapov and Polyushkina [2010] have shown diurnal magnetic field spectra in a geostationary orbit, whose peaks are similar to those in the SW magnetic field spectrum (Figure 5). The authors assumed that the MHD waves penetrated directly into the magnetosphere. Wright and Rickard [1995] have demonstrated the possibility of exciting magnetospheric waves with discrete frequencies (the so-called magic frequencies) by the broadband oscillation spectrum in SW. This unusual feature can be explained by the model of FMSwave resonator forming in the near plasma sheet [Mazur, Leonovich, 2006]. It is explained by the magnetospheric wave resonator model ascribing dominant resonance frequencies to eigenoscillations of the magnetosphere as a whole.

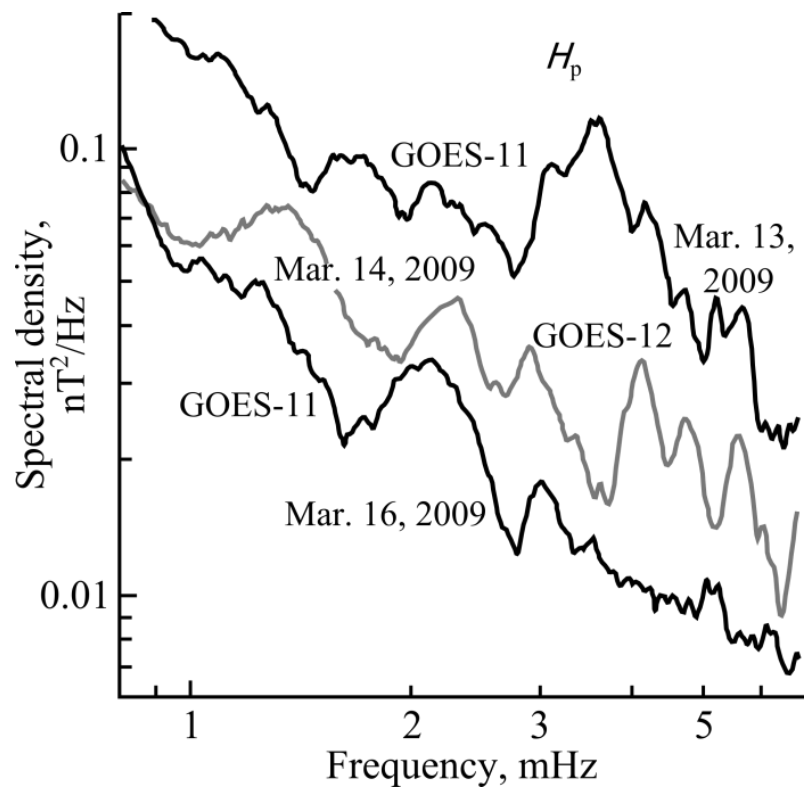

Figure 5. Three diurnal spectra of oscillations in magnetic field $H p$ component 
Some authors suggest that these spectrum peaks prevail in SW pressure fluctuations rather than necessarily originate within the magnetosphere [Kepko et al., 2002; Viall et al., 2009]. Mishin et al. [2003] have identified the dependence of periods of pulsations caused by SW pressure fluctuations as the fourth power of the distance to the subsolar point of the magnetosphere.

Another possible manifestation of the mechanisms of pulsation energy transfer from the solar atmosphere to Earth's magnetosphere can be observed in the 0.1-5 $\mathrm{Hz}$ frequency range (Pc1-2 pulsations): the so-called serpentine emission (SE) [Guglielmi, Dovbnya, 1973] is recorded in polar caps and represents ion-cyclotron waves, which feature deep frequency modulation. The assumption about the external magnetospheric source of these oscillations was made on the basis of observations showing characteristic five-minute modulation of oscillations, which is probably associated with five-minute oscillations recorded in the solar photosphere [Guglielmi et al., 2015]. It is, however, obvious that generation of this emission is not well understood and the assumption that these oscillations penetrate from SW into the magnetosphere needs further theoretical justification [Dovbnya, Potapov, 2018].

In some cases, the phenomena recorded in nearEarth space can be interpreted as a response to the oscillations occurring on the Sun. Hayes et al. [2017] have examined the example when electron density variations in the ionosphere echo quasiperiodic pulsations in $\mathrm{X}$ rays of solar flares. The period of the X-ray pulsations was $\sim 20 \mathrm{~min}$; the delay of the ionospheric response, $\sim 90$ s. While this case could not be a typical example of earthward oscillation energy transfer by MHD waves, it shows how solar oscillations can generate ionospheric oscillations. Waves in near-Earth space can also be caused by variations of SW parameters. Zolotukhina [2009] has shown that SW pressure fluctuations can excite magnetospheric oscillations.

Comparing small-scale phenomena on the Sun with specific ionospheric and magnetospheric responses to them is a difficult task. Potapov et al. [2013] have analyzed spectral oscillations at bases of coronal holes and spectral peaks of interplanetary magnetic field variations (Figure 6). They tried to analyze to what extent solar photospheric oscillations contribute to SW oscillations in Earth's orbit.

Chromospheric line profiles exhibit pronounced half-width oscillations, which can be attributed to Alfvén waves. Spectral peaks of these oscillations range from 2 to $6 \mathrm{mHz}$. In SW, which feature Alfvén waves, these peaks can also be detected, although, in general, SW spectra have a more complex frequency distribution and irregular behavior.

Characteristics of oscillations observed in different media indicate that Alfvén waves generated in the solar chromosphere and transported by SW can provide the energy for the Alfvén resonance of magnetospheric field lines.

\section{CONCLUSIONS}

The brief analysis of observations made at ISTP SB RAS has shown that oscillation spectra in the solar chromosphere and Earth's magnetosphere have much in

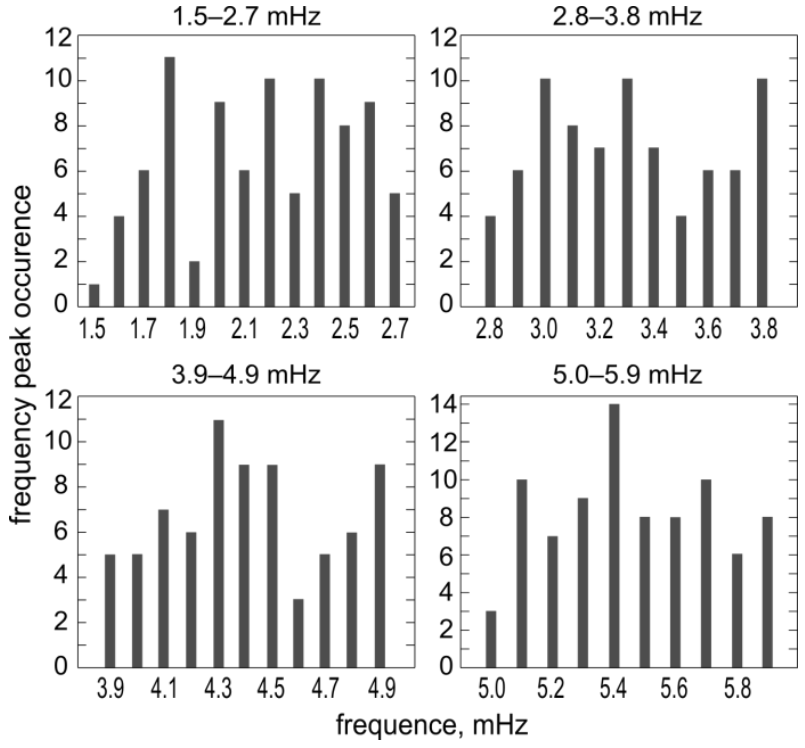

Figure 6. Distribution of peaks in different frequency ranges observed in high-speed SW streams between 1999 and 2006. The histograms are based on data obtained by Potapov et al. [2013]

common. This is another argument for joining the two formally distant areas of research. It is hoped that in the near future these areas will be enriched with new ideas and approaches to the problems under study [Nakariakov et al., 2016a].

The connection between solar and magnetospheric oscillations is generally searched for in the five-minute range, which is obviously due to the prevalence of fiveminute oscillations in the solar atmosphere. The spectra of the observed solar and magnetospheric oscillations are, however, much richer.

We think that much attention should be paid to threeminute oscillations common in the solar chromosphere and to $10-15$ min oscillations observed both in the Doppler velocity and in different solar structures.

The work was performed with budgetary funding of Basic Research program II.16.1.3, II.16.3.2, and II.16.3.3. The results were obtained using the equipment of Center for Common Use "Angara" http://ckprf.ru/ckp/3056/.

\section{REFERENCES}

Anfinogentov S.A., Nakariakov V.M., Nisticò G. Decayless low-amplitude kink oscillations: a common phenomenon in the solar corona? Astron. Astrophys. 2015, vol. 583, id. A136. DOI: 10.1051/0004-6361/201526195.

Anfinogentov S., Sych R., Prosovetsky D. Induced MHD oscillations of fine loop structures located in coronal hole. arXiv: $1011.4350,11 / 2010$.

Chelpanov, A.A., Kobanov N.I., Kolobov D.Yu. Characteristics of oscillations in magnetic knots of solar faculae. Astron. Rep. 2015, vol. 59, pp. 968-973.

Chelpanov, A., Kobanov, N., Chupin, S. Search for the observational manifestations of torsional alfvén waves in solar faculae. Central European Astrophys. Bull. 2016a, vol. 40, pp. 29-34.

Chelpanov, A.A., Kobanov, N.I., Kolobov, D.Yu. Influence of the magnetic field on oscillation spectra in solar faculae. Solar Phys. 2016b, vol. 291, pp. 3329-3338.

Cranmer S.R., van Ballegooijen A.A. On the generation, 
propagation, and reflection of Alfvén waves from the solar photosphere to the distant heliosphere. The Astrophys. J. Supplement Ser. 2005, vol. 156, iss. 2. pp. 265-293.

De Pontieu B., McIntosh S., Martinez-Sykora, et al. Why is non-thermal line broadening of spectral lines in the lower transition region of the Sun independent of spatial resolution? The Astrophys. J. Lett. 2015, vol. 799, L12.

Dmitrienko I.S., Space-time structure of Alfvén resonant disturbances generated by transversally localized FMA wave. Geomagnetism and Aeronomy. 2010, vol. 50, iss. 8., pp. 1025-1034.

Dovbnya B.V., Potapov A.S. The frequency modulation of serpentine emission as compared to the set of the known periodicities of solar oscillations. Izvestiya, Physics of the Solid Earth. 2018, vol. 54. iss. 5, pp. 680-687. DOI: 10.1134/S1069351318050051.

Greenstadt E.W., Mellott M.M., McPherron, Russell C.T., Singer H.J., Knecht D.J. Transfer of pulsation-related wave activity across the magnetopause - observations of corresponding spectra by ISEE-1 and ISEE-2. Geophys. Res. Lett. 1983, vol. 10, pp. 659-662.

Guglielmi A.V. The magnetosphere and interplanetary medium diagnostics from observation of geomagnetic pulsations. Doklad na Vsesoyuznom seminare po fizike magnitosfery [Report at the National Conference on Physics of the Magnetosphere]. Borok, 1972. (In Russian).

Guglielmi A.V., Dovbnya B.V. Hydromagnetic emission of interplanetary plasma. Pis'ma v ZhETF [Pis'ma v Zhurnal Èksperimental'noi i Teoreticheskoi Fiziki]. 1973, vol. 18, iss. 10, pp. 601-604. (In Russian).

Guglielmi, A. V., Troitskaya V.A. Geomagnitnye pulsatsii $i$ diagnostika atmosfery [Geomagnetic pulsations and the atmosphere diagnostics]. Moscow, Nauka Publ., 1973, 208 pp. (In Russian).

Guglielmi A.V., Potapov A.S. On pecularities of MHD wave field in inhomogeneous plasma. Issledovaniya po geomagnetizmu, aeronomii i fizike Solnta [Res. on Geomagnetism, Aeronomy and Solar Phys.]. 1984, iss. 70, pp. 149157. (In Russian).

Guglielmi A., Potapov A., Dovbnya B. Five-minute solar oscillations and ion-cyclotron waves in the solar wind. Solar Phys. 2015, vol. 290, iss. 10, pp. 3023-3032. DOI: 10.1007/s11207-015-0772-2.

Hayes L.A., Gallagher P.T., McCauley, Dennis B.R, Ireland J., Inglis A. Pulsations in the Earth's lower ionosphere synchronized with solar flare emission. J. Geophys. Res. Space Phys. 2017, vol. 122. pp. 9841-9847.

Kepko L., Spence H.E., Singer H.J. ULF waves in the solar wind as direct drivers of magnetospheric pulsations. $G e-$ ophys. Res. Lett. 2002, vol. 29 (8), pp. 39-1-39-4. URL http://dx.doi.org/10.1029/2001GL014405.

Kessel R.L. Solar wind excitation of Pc5 fluctuations in the magnetosphere and on the ground. J. Geophys. Res.: Space Phys. 2008, vol. 113, A04202..

Kobanov N.I. Narrowband spatial filtering in differential measurements of the line-of-sight velocity of the solar atmosphere. Astron. Astrophys. 1985. vol. 143. pp. 99-101.

Kobanov N.I. The properties of velocity oscillations in vicinities of sunspot penumbra. Solar Phys. 2000a, vol. 196, pp. 129-135.

Kobanov N.I. Properties of oscillations in sunspot penumbras. Astronomy Rep. 2000b, vol. 44, iss. 3, pp. 202-208.

Kobanov N.I. Measurement of the differential ray velocity and longitudinal magnetic field on the sun with CCD photodetectors: Part I. Modulationless method. Instruments and Experimental Techniques. 2001, vol. 44, pp. 524-529.

Kobanov N.I., Makarchik D.V., Sklyar A.A. Photospheric and chromospheric oscillations in the base of coronal holes.
Solar Phys. 2003, vol. 217, pp. 53-67. DOI: 10.1023/A:1027301101788.

Kobanov N.I., Pulyaev V.A. Photospheric and chromospheric oscillations in solar faculae. Solar Phys. 2007, vol. 246, pp. 273-279.

Kobanov N.I., Sklyar A.A. Periodic processes and plasma motions in solar coronal holes. Astron. Rep. 2007, vol. 51, pp. 773-779.

Kobanov N.I., Kustov A.S., Pulyaev V.A., Chupin S.A. The role of faculae in wave-energy transfer to upper layers of the solar atmosphere: Observations. Astron. Rep. 2011, vol. 55, pp. 532-540.

Kobanov N., Kolobov D., Kustov A., Chupin S., Chelpanov A. Direct measurement results of the time lag of LOS-velocity oscillations between two heights in solar faculae and sunspots. Solar Phys. 2013a, vol. 284, pp. 379-396.

Kobanov N.I., Chelpanov A.A., Kolobov D.Yu. Oscillations above sunspots from the temperature minimum to the corona. Astron. Astrophys. 2013b, vol. 554, pp. A146.

Kobanov N., Kolobov D., Chelpanov A. Oscillations above sunspots and faculae: height stratification and relation to coronal fan structure. Solar Phys. 2015, vol. 290, pp. 363-380.

Kolobov D.Yu., Chelpanov A.A., Kobanov N.I. Peculiarity of the oscillation stratification in sunspot penumbrae. Solar Phys. 2016, vol. 291, pp. 3339-3347.

Lee, L. C., Albano, R. K., Kan, J. R., Kelvin - Helmholtz instability in the magnetopause-boundary layer region. $J$. Geophys. Res.: Space Phys. 1981, vol. 86 (A1), pp. 54-58. URL: http://dx.doi.org/10.1029/JA086iA01p00054.

Leonovich, A., Mazur, V., Kozlov, D., MHD-waves in the geomagnetic tail: A review. Solnechno-zemnaya fizika [SolarTerr. Phys.] 2015, vol. 1, no. 1, pp. 4-22. (In Russian).

Lipko Y.V., Pashinin A.Y., Rahkmatulin R.A. Ionospheric manifestations of geomagnetic pulsations at high latitudes. Eighth International Symposium on Atmospheric and Ocean Optics: Atmospheric Phys. 2002, vol. 4678, pp. 485-490.

Mazur V.A. Resonance excitation of the magnetosphere by hydromagnetic waves incident from solar wind. Plasma Phys. Rep. 2010, vol. 36, pp. 953-963.

Mazur V.A., Leonovich A.S. ULF hydromagnetic oscillations with the discrete spectrum as eigenmodes of MHDresonator in the near-Earth part of the plasma sheet. Annales Geophysicae. 2006, vol. 24, pp. 1639-1648. DOI: 10.5047/eps.2012.07.006.

Mishin V.V. On the MHD instability of the Earth's magnetopause and its geophysical effects. Planetary and Space Sci. 1981, vol. 29, iss. 3, pp. 359-363.

Mishin V.V. Accelerated motions of the magnetopause as a trigger of the Kelvin - Helmholtz instability. J. Geophys. Res. 1993, vol. 98 (A12), pp. 21365-21371.

Mishin V.V., Tomozov V.M. Kelvin - Helmholtz instability in the solar atmosphere, solar wind and geomagnetosphere. Solar Phys. 2016, vol. 291, pp. 3165-3184. DOI: 10.1007/s11207-016-0891-4.

Mishin V., Parkhomov V., Pashinin A. Geomagnetic pulsations caused by the magnetopause oscillations (comparison of spacecraft and geomagnetic observations). Adv. Space Res. 2003, vol. 31 (5), pp. 1177-1182.

Nakariakov V.M., Verwichte E. Coronal waves and oscillations. Living Rev. in Solar Phys. 2005, vol. 2, pp. 3. DOI: 10.12942/lrsp-2005-3.

Nakariakov V.M., Anfinogentov S.A., Nisticò G., Lee D.-H. Undamped transverse oscillations of coronal loops as a selfoscillatory process. Astron. Astrophys. 2016a, vol. 591, id. L5. DOI: 10.1051/0004-6361/201628850.

Nakariakov V.M., Pilipenko V., Heilig B., et al. Magnetohydrodynamic oscillations in the solar corona and Earth's magnetosphere: towards consolidated understanding. Space 
Sci. Rev. 2016b, vol. 200, pp. 75-203.

Nosé M., Iyemori T., Sugiura M., et al. A strong dawn/dusk asymmetry in Pc5 pulsation occurrence observed by the DE-1 satellite. Geophys. Res. Lett. 1995, vol. 22 (15), pp. 2053-2056. URL: http://dx.doi.org/10.1029/95GL01794.

Olson J.V., Rostoker G. Longitudinal phase variations of Pc4-5 micropulsations. J. Geophys. Res. 1978, vol. 83, pp. 2481-2488.

Potapov A.S., Mazur V.A. Pc3 pulsations: From the source in the upstream region to Alfven resonances in the magnetosphere. Theory and observations. Geophys. Monograph Ser. 1994, vol. 81, pp. 135-145.

Potapov A.S., Polyushkina T.N. Experimental evidence for direct penetration of ULF waves from the solar wind and their possible effect on acceleration of radiation belt electrons. Geomagnetism and Aeronomy. 2010, vol. 50, pp. 950-957.

Potapov A.S., Polyushkina T.N., Pulyaev V.A. Observations of ULF waves in the solar corona and in the solar wind at the Earth's orbit. J. Atmos. Solar-Terr. Phys. 2013, vol. 102, pp. 235-242.

Rakhmatulin R.A. On a possibility of existence of two and more sources $\mathrm{Pi} 2$ pulsations in the magnetosphere. International Association of Geomagnetism and Aeronomy IAGA-11. Scientific Assembly. Sopron (Hungary), 23-30 Aug 2009. P. 1212. Available from URL: http://www.iaga2009sopron.hu.

Rakhmatulin R.A., Pashinin A.Yu., Hayashi K. Observation of global $\mathrm{Pi} 2$ pulsations in mid-latitudes during auroral substorms. 5th International Conference on Substorms. St. Petersburg, Russia, 16-20 May, 2000. Proc. Noordwijk. European Space Agency, ESA SP. 2000, V. 443. P. 561-564.

Shestov S.V., Nakariakov V.M., Ulyanov A.S., Reva A.A., Kuzin S.V. Nonlinear evolution of short-wavelength torsional Alfvén waves. The Astrophys. J. 2017, vol. 840, iss. 2, id. 64 . DOI: 10.3847/1538-4357/aa6c65.

Stephenson J.A.E., Walker A.D.M. HF radar observations of Pc5 ULF pulsations driven by the solar wind. Geophys. Res. Lett. 2002, vol. 29 (9), pp. 8-1-8-4. URL: http://dx.doi.org/10.1029/2001GL014291.

Sych R., Nakariakov V., Karlicky M., Anfinogentov S. Relationship between wave processes in sunspots and quasiperiodic pulsations in active region flares. Astron. Astrophys. 2009, vol. 505, iss. 2. pp. 791-799.

Sych R., Zaqarashvili T.V., Nakariakov V.M., et al. Frequency drifts of 3-min oscillations in microwave and EUV emission above sunspots. Astron. Astrophys. 2012, vol. 539, id. A23. DOI: 10.1051/0004-6361/201118271.

Takahashi K., Ukhorskiy A.Y. Solar wind control of Pc5 pulsation power at geosynchronous orbit. J. Geophys. Res.: Space Phys. 2007, vol. 112 (A11), a11205. URL: http://dx.doi.org/10.1029/2007JA012483.

Takahashi K., Ukhorskiy A.Y. Timing analysis of the relationship between solar wind parameters and geosynchronous Pc5 amplitude. J. Geophys. Res.: Space Phys. 2008, vol. 113 (A12), a12204. URL: http://dx.doi.org/10.1029/2008JA013327.

Troitskaya V.A., Plyasova-Bakunina T.A., Guglielmi A.V. Correlation between Pi2-4 pulsations and the interplanetary magnetic field. Doklady AN SSSR [Doklady AS USSR]. 1971, vol. 197, no. 6, pp. 1312-1314.

Viall N.M., Kepko L., Spence H.E. Relative occurrence rates and connection of discrete frequency oscillations in the solar wind density and dayside magnetosphere. J. Geophys. Res.: Space Phys. 2009, vol. 114 (A1), a01201. URL: http://dx.doi.org/10.1029/2008JA013334.

Wright A.N., Rickard G.J. ULF pulsations driven by magnetopause motions: Azimuthal phase characteristics. J. Geophys. Res.: Space Phys. 1995, vol. 100 (A12), pp. 2370323710. URL: http://dx.doi.org/10.1029/95JA01765.

Yumoto K. External and internal sources of low-frequency MHD waves in the magnetosphere. A. Review. J. Geomag- netism and Geoelectricity. 1988, vol. 40, iss. 3, pp. 293-311.

Zolotukhina N.A. Resonance properties of Psi5/Psc5 in geostationary orbit. Geomagnetism and Aeronomy. 2009, vol. 49, pp. 438-449.

Zolotukhina N.A. Polekh N.M., Rakhmatulin R.A., et al. Geophysical effects of the interplanetary magnetic cloud on October 18-19, 1995 as deduced from observations at Irkutsk. J. Atmos. Solar-Terr. Phys. 2000, vol. 62, pp. 737-749.

Zubkova A.V., Kobanov N.I., Sklyar A.A., Kostyk R.I., Shchukina N.G. Periodic variations of the H-alpha profile width in the chromosphere of coronal holes as a possible indicator of Alfvén waves. Astronomy Lett. 2014, vol. 40, pp. 222-229.

How to cite this article

Chelpanov A.A., Chelpanov M.A., Kobanov N.I., Sotnikova R.T. Comparing the main oscillation characteristics in the solar chromosphere and magnetosphere based on studies made at ISTP SB RAS. Solarterrestrial physics. 2018. Vol. 4. Iss. 4. P. 12-18. DOI: 10.12737/stp44201802 . 